\title{
Far-red Light Interacts with Plant Density to Change Photosynthate Allocation of Cucumber Seedlings and Their Subsequent Early Growth after Transplanting
}

\author{
Toshio Shibuya \\ Graduate School of Life and Environmental Sciences, Osaka Prefecture \\ University, 1-1 Gakuen-cho, Naka-ku, Sakai, Osaka 599-8531, Japan
}

Mizuki Tsuchida

School of Life and Environmental Sciences, Osaka Prefecture University, 1-1 Gakuen-cho, Naka-ku, Sakai, Osaka 599-8531, Japan

Ryosuke Endo and Yoshiaki Kitaya

Graduate School of Life and Environmental Sciences, Osaka Prefecture University, 1-1 Gakuen-cho, Naka-ku, Sakai, Osaka 599-8531, Japan

Additional index words. LED, light competition, light quality, phytochrome, shoot elongation, transplant production

\begin{abstract}
The light competition in dense plant stands may be disadvantageous in transplant production because competition stimulates stem elongation and can reduce photosynthate allocation to leaves; this, in turn, may reduce the early growth rate after transplanting. In this study, we focused on how the proportion of far-red (FR) light affected

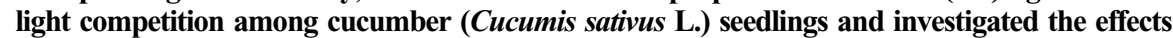
of the plant density $\times$ FR interaction on photosynthate allocation and subsequent early growth after transplanting. Seedlings at the cotyledon stage were planted into plug trays at densities ranging from 109 to 1736 plants $/ \mathrm{m}^{2}$; then they were grown for 4 days under lightemitting diode (LED) light containing FR light (FR+) at approximately the same red-to-FR ratio as in sunlight (1.2) or under light containing no FR (FR-). The higher density significantly stimulated stem elongation under both FR+ and FR-, but the effect was small under FR-; this indicates that light competition in the dense stands was inhibited by reducing FR light. The higher plant density significantly increased photosynthate allocation to the stem and decreased allocation to the leaves under both FR+ and FR-; however, again, the effect was smaller under FR-. After transplanting the seedlings to pots, early growth decreased in the seedlings that allocated less photosynthate to their leaves. Our results indicate that light with reduced FR can mitigate the disadvantageous photosynthate allocation of transplants and the reduction of early growth after transplanting that are likely to occur as a result of light competition at high plant density.
\end{abstract}

During transplant production, young plants are generally grown densely to improve the productivity per unit growing area (Marr and Jirak, 1990). Growing plants at high density stimulates stem elongation due to increased competition for light (Nagashima and Terashima, 1995; Vermeulen et al., 2008; Weiner and Thomas, 1992); this is mainly caused by a shade-avoidance response that is stimulated by an increase in the ratio of FR light to red light within the canopy

Received for publication 25 May 2020. Accepted for publication 18 June 2020 .

Published online 30 July 2020.

This research was supported by a Japan Society for the Promotion of Science Grant-in-Aid for Scientific Research (B) (KAKENHI 18H02307).

T.S. is the corresponding author. E-mail: shibuya@ envi.osakafu-u.ac.jp.

This is an open access article distributed under the CC BY-NC-ND license (https://creativecommons. org/licenses/by-nc-nd/4.0/).
(Ballaré et al., 1994; Franklin, 2008; Smith and Whitelam, 1997) and by the reflected FR light from neighboring plants (Ballaré et al., 1987, 1990). This competition can benefit dense plant stands in natural ecosystems by producing more uniform plants stands with an improved mechanical structure (Nagashima and Hikosaka, 2011). However, this optimization may be disadvantageous in transplant production, during which plants grown at high plant density must be separated for transplanting; the enhanced stem elongation reduces the allocation of photosynthate to leaves as a result of competition between stems and leaves as sinks (Casal et al., 1987; Poorter et al., 2012). This, in turn, may reduce the early growth rate after transplanting, which is one of the main parameters of transplant quality, due to the decreased photosynthetic tissue. In addition, the excessive elongation may reduce the resistance to mechanical stresses (Latimer and Mitchell, 1988). Therefore, there may be trade-offs between the productivity of transplants per unit growing area and the quality of the individual transplants.

In this study, we focused on the interaction of plant density with the proportion of FR light and the effects of this interaction on stem elongation due to light competition and subsequent competition between stems and leaves for photosynthate allocation. Previous research suggests that decreasing the proportion of FR light can reduce light competition of dense plant stands (Shibuya et al., 2013, 2016b). We hypothesized that this would mitigate the effects of decreased photosynthate allocation to the leaves, which may be disadvantageous for subsequent early growth, even when transplants are grown at a high plant density. To test this hypothesis, we investigated the effects of the plant density $\times$ FR interaction on photosynthate allocation and subsequent early growth of cucumber ( $\mathrm{CuCu}$ mis sativus L.) seedlings after transplanting.

\section{Materials and Methods}

Except when otherwise noted, each experiment was performed seven times (i.e., $\mathrm{n}=$ 7). The values represent the mean of these replicates.

Preparation of plant stands. Cucumber (cultivar Hokushin) seeds were sown in plug trays (cell size, $20 \mathrm{~mm} \times 20 \mathrm{~mm}$; depth, $40 \mathrm{~mm}$ ) containing vermiculite medium and grown in a growth chamber for $6 \mathrm{~d}$ until the cotyledons had expanded. The air temperature, relative humidity, and $\mathrm{CO}_{2}$ concentration in the growth chamber were maintained at $28{ }^{\circ} \mathrm{C}, 55 \%$, and $400 \mu \mathrm{mol} \cdot \mathrm{mol}^{-1}$, respectively. Illumination was supplied by fluorescent lamps (FHF32EXNH; Panasonic Co., Kadoma, Japan) at a photosynthetic photon flux density $(P P F D)$ of $300 \mu \mathrm{mol} \cdot \mathrm{m}^{-2} \cdot \mathrm{s}^{-1}$. The trays were continuously immersed to a depth of 5 to $10 \mathrm{~mm}$ in nutrient solution (Atype recipe of OAT Agrio Co. Ltd., Tokyo, Japan). Six days after seeding (DAS), plant stands with different plant densities were prepared by transplanting the seedlings to new plug trays with the same cell size at densities of 109 plants $/ \mathrm{m}^{2}$ (low density), 886 plants $/ \mathrm{m}^{2}$ (medium density), or 1736 plants $/ \mathrm{m}^{2}$ (high density) (Fig. 1). The typical plant density for producing cucumber transplants is 388 to 992 plants $/ \mathrm{m}^{2}$ (calculated from the number of cells per tray) (Drost, 2014), which is within the range of densities used in this study. The seedlings under low plant density were planted in single cells separated from the trays. The seedlings under medium plant density were planted in the plug trays $(7 \times 7$ cells per tray) in a staggered pattern with their cotyledons in parallel lines but not overlapping. At the high plant density, the seedlings were placed in the plug trays $(6 \times 6$ cells per tray) with their cotyledons in parallel lines; however, in this case, each seedling had one of its cotyledons covered by a cotyledon from the next seedling. Wire netting was installed around the medium-density and high-density plant stands to prevent the outermost seedlings from expanding beyond the plug tray. 
Low density
$\left(109\right.$ plants $\left./ \mathrm{m}^{2}\right)$

उथ 8
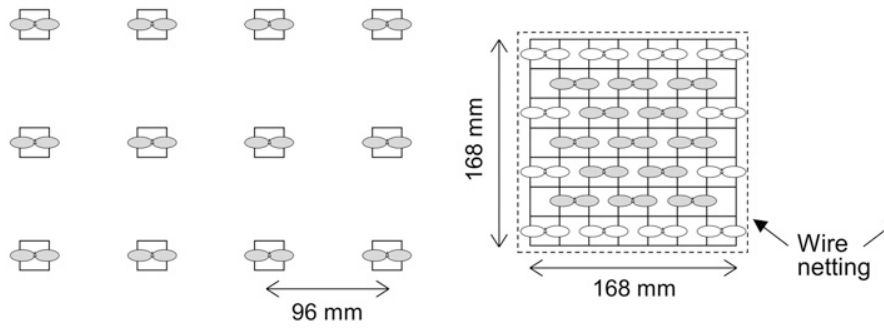

$168 \mathrm{~mm}$
High density (1736 plants $\left./ \mathrm{m}^{2}\right)$

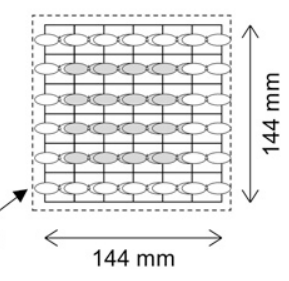

Fig. 1. Positions of the seedlings in each plant density treatment. The seedlings in marginal areas (white cotyledons) were not measured in the medium- and high-density treatments.

Lighting treatment. The plant stands were grown in the growth chamber for $4 \mathrm{~d}$ (from 6 to 10 DAS) under FR+ or FR-. The illumination was provided by LED panels (LED SUN LIGHT Z4; Taiwan Hipoint Co., Kaohsiung, Taiwan) containing blue, green, red, and (in the FR + treatment) FR light sources (Fig. 2). The blue light (400-500 nm) accounted for $36 \%$ of the photosynthetically active radiation $(400-700 \mathrm{~nm})$, green $(500-600 \mathrm{~nm})$ accounted for $20 \%$, and red (600-700 nm) accounted for $44 \%$. The red-to-FR ratio in the FR+ treatment was 1.2 , which is the same proportion in sunlight. The $P P F D$ at the leaf surface was maintained at $300 \mu \mathrm{mol} \cdot \mathrm{m}^{-2} \cdot \mathrm{s}^{-1}$ during plant growth by adjusting the distance between the lamps and the canopy surface daily. The light:dark period was 16:8 h throughout the experiment. Four days after starting treatment (i.e., at 10 DAS), some of the seedlings in each treatment group were sampled to measure the growth parameters described here. The remaining seedlings (five seedlings for each treatment) were transplanted to plastic pots (diameter, $90 \mathrm{~mm}$; height, $76 \mathrm{~mm}$ ) containing vermiculite medium and grown without mutual shading under FR + for an additional $4 \mathrm{~d}$ (i.e., from 10 to 14 DAS). Because the hypocotyls of the seedlings could not support their shoot, the plants, which had been grown at medium and high density under FR+, were supported by stakes. The environmental conditions, except light quality, were the same as those before transplanting.

The spectra of the two illumination treatments were measured using an SS-110 spectroradiometer (Apogee Instruments, Logan,

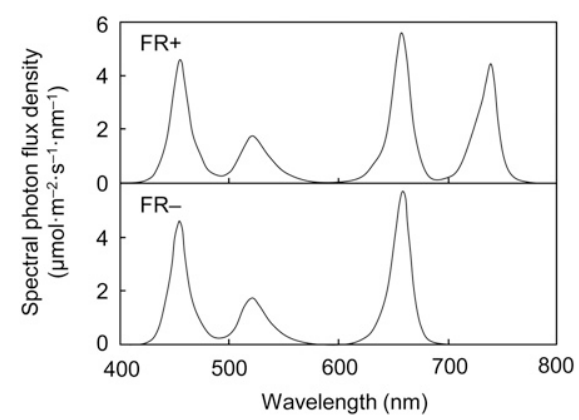

Fig. 2. Spectral photon flux of the light sources containing far-red (FR) at approximately the same proportion as that of sunlight $(\mathrm{FR}+)$ and light containing no FR (FR-).
UT), and their phytochrome photostationary state (PSS), which represents the ratio of active phytochrome to total phytochrome, was calculated according to the method of Sager et al. (1988). In addition, we estimated the PSS based on the FR+ and FR- illuminations transmitted through a cucumber leaf.

Measurements of growth parameters. Four days after the start of treatment (at 10 DAS), we destructively sampled 7, 8, and 11 seedlings from the low-, medium-, and high-density treatments, respectively. We measured the dry mass (DM) of the leaves, stem (including hypocotyl and petioles), and roots after oven-drying for $72 \mathrm{~h}$ at $80{ }^{\circ} \mathrm{C}$, and we measured the stem length and leaf area. The seedlings at the edges of the trays were not measured in the mediumand high-density treatments to eliminate edge effects. The dry matter production of plant stands per unit growing area $\left(\mathrm{g} \cdot \mathrm{m}^{-2}\right)$ was determined by multiplying the total DM ( $g$ per plant) by the plant density (plants $/ \mathrm{m}^{2}$ ). We calculated the ratios of the leaf, stem, and root DM to the total DM to represent the photosynthate allocation to each organ. Stem length per unit of shoot DM (leaves plus stem) was calculated to standardize the stem elongation. The ability to self-support was assessed by dividing the height of seedlings after separating from the plant stands by the height before separation to represent the mechanical strength of the shoots. The self-support measurement only used four replicates. We determined the leaf area index (LAI) of the plant stands at $4 \mathrm{~d}$ after the start of treatment (i.e., at 10 DAS) by dividing the total leaf area of the plant stands by the growing area.

Four days after transplanting to the pots (i.e., at 14 DAS), we measured the DM and leaf area of the remaining five seedlings in each treatment group. We calculated the relative growth rate $\left(\mathrm{RGR}, \mathrm{d}^{-1}\right)$, net assimilation rate (NAR, $\mathrm{g} \cdot \mathrm{m}^{-2} \cdot \mathrm{d}^{-1} \mathrm{DM}$ basis), and leaf area ratio $\left(\mathrm{LAR}, \mathrm{m}^{2} \cdot \mathrm{g}^{-1}\right.$ ) after transplanting to the pots by using the following equations (Hunt et al., 2002; Radford, 1967):

$$
\begin{gathered}
\mathrm{RGR}=\frac{\ln W_{2}-\ln W_{1}}{t_{2}-t_{1}}=\mathrm{NAR} \times \mathrm{LAR} \\
\mathrm{NAR}=\frac{W_{2}-W_{1}}{A_{2}-A_{1}} \times \frac{\ln A_{2}-\ln A_{1}}{t_{2}-t_{1}}
\end{gathered}
$$

$$
\mathrm{LAR}=\frac{A_{2}-A_{1}}{\ln A_{2}-\ln A_{1}} \times \frac{\ln W_{2}-\ln W_{1}}{W_{2}-W_{1}}
$$

where $W_{1}$ and $W_{2}$ are the total DM (g per plant) at times $t_{1}$ and $t_{2}$ (at 10 and 14 DAS, respectively) and $A_{1}$ and $A_{2}$ are the corresponding total leaf areas ( $\mathrm{m}^{2}$ per plant).

Two days after transplanting to the pots (i.e., at 12 DAS), we measured the net photosynthetic rate $\left(\mathrm{P}_{\mathrm{n}}\right)$ of the first true leaves of four seedlings in both the low- and high-density treatments with an LI-6400 photosynthesis system (LI-COR Inc., Lincoln, NE) under growing light condition $(\mathrm{FR}+)$. The $\mathrm{P}_{\mathrm{n}}$ of medium-density treatment was not measured due to time constraints. The measurement of photosynthesis only used four replicates.

Statistical analysis. The effects of the plant density $\times$ FR interaction on each parameter were determined by the means of a two-way analysis of variance (ANOVA). When the ANOVA result was significant, we identified significant differences between the treatments by using the Tukey-Kramer test (with significance at $P<0.05$ ). The relationships between photosynthate allocation (ratios of the leaf, stem, and root DM to total DM) at transplanting and the growth analysis parameters (RGR, NAR, and LAR) after transplanting to the pots and between dry matter production of plant stands per unit growing area and RGR after transplanting to the pots were analyzed with Pearson's product-moment correlation coefficient $(r)$ for the whole dataset (i.e., all six treatments in each replicate combined). All analyses were performed with the Statcel 2 software (OMS Publishing, Inc., Tokorozawa, Japan).

\section{Results and Discussion}

Stem elongation. The stem elongation was significantly stimulated by the higher plant density and by FR+ (Fig. 3A). The stem length had a significant plant density $\times$ FR interaction; the effect of plant density was stronger under FR + . In the FR+ treatment, stem length did not differ significantly between the medium and high plant densities. This is probably because the decreased photosynthate production caused by mutual shading limited stem elongation at the high plant density. When we standardized stem elongation for plant size, stem elongation (represented by the stem length per unit shoot DM) was greater at the high plant density than at the medium density (Fig. 3B). The stem length had a significant plant density $\times$ FR interaction; the effect of plant density was greater under FR+ than under FR-. The increased stem elongation at higher density under FR+ occurred because the increased mutual shading increased the proportion of FR in the transmitted light and increased light competition through the decreased proportion of active phytochrome (Ballaré et al., 1991; Franklin, 2008). The PSS determined for the incident light spectrum of $\mathrm{FR}+$ and the 

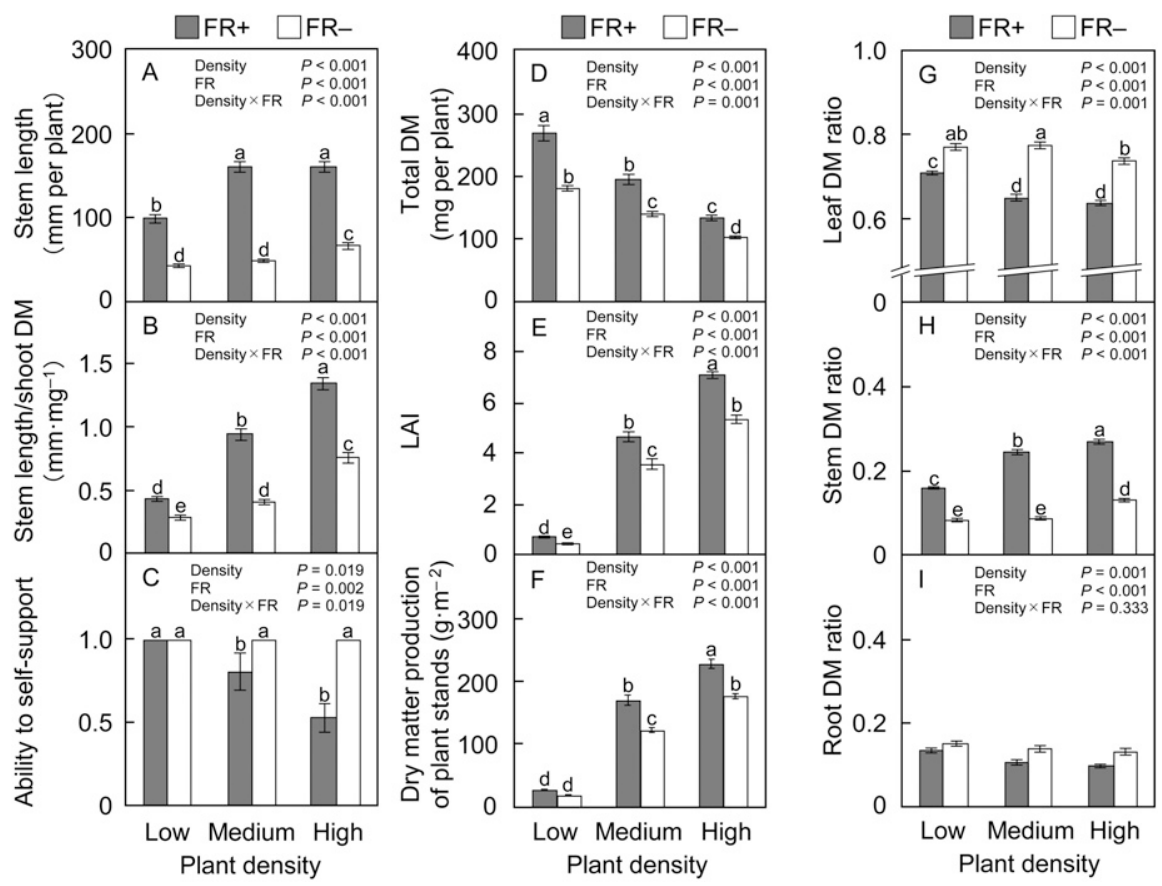

Fig. 3. Effects of plant density and far-red (FR) treatment (FR+, FR light similar to sunlight; FR -, no FR light) on the (A) stem length, (B) stem length/shoot dry mass (DM), (C) ability to self-support, (D) total DM per individual plant, (E) leaf area index (LAI), (F) DM production of plant stands per unit growing area, and the ratios of $(\mathbf{G})$ leaf, $(\mathbf{H})$ stem, and (I) root DM to total DM of the cucumber seedlings at $4 \mathrm{~d}$ after the start of treatment (10 d after seeding). The ability to self-support was assessed by dividing the height of seedlings after separating from the plant stands by the height before separation. Data are the average \pm SE of seven and four replicates for stem length/shoot DM $(n=7)$ and ability to self-support $(\mathrm{n}=4)$, respectively. Bars for a parameter labeled with the same letter are not significantly different at $P<0.05$ (analysis of variance followed by the Tukey-Kramer test).

spectrum of FR + transmitted through a leaf were 0.72 and 0.56 , respectively.

However, the effect of plant density on the standardized stem elongation in the FR- treatment was less than that under FR+, especially at low to medium plant densities (Fig. 3B). This is because light competition was inhibited by reducing the amount of FR light (Shibuya et al., 2013, 2016a). As in the FR+ treatment, the standardized stem elongation in the FRtreatment was greater at the higher plant density than at the medium density, indicating that higher plant density can stimulate shade-avoidance responses even under FR- through factors other than a decreased proportion of active phytochrome, for example, due to decreased light intensity (Vandenbussche et al., 2003). The PPS of shaded leaves under FR- light was not changed by light transmission through leaves in this experiment. The PSS determined for the incident light spectrum of FR- and the spectrum of FR - transmitted through a leaf were 0.87 and 0.88 , respectively.

The ability to self-support in the FR + treatment decreased significantly as plant density increased, whereas the degree under the FRtreatment was not significantly affected by plant density (Fig. 3C). This indicates that the mechanical strength of individual seedlings decreased when light competition was increased by growing the plants at high density under $\mathrm{FR}+$, but that this reduction was unlikely to occur under light with reduced FR.
Total dry mass and leaf area. The total DM per individual plant was significantly less under FR - than under FR+ at all densities, and it decreased as the plant density increased (Fig. 3D). The smaller DM under FR - is probably due to the inhibition of leaf enlargement that occurs under light with reduced FR (Park and Runkle, 2017; Shibuya et al., 2016a). In addition, photosynthesis per unit leaf area may be reduced under FR- because FR light can increase the photosynthetic efficiency of light with shorter wavelengths (Kono et al., 2020; Zhen and Bugbee, 2020; Zhen and van Iersel, 2017). We found a statistically significant plant density $\times$ FR interaction with total DM per individual plant; the effect of the plant density on total DM was less under FR- than under FR+. The difference in total DM per individual plant between $\mathrm{FR}+$ and FR- decreased with the increasing plant density. This is partly because the decreased leaf enlargement under FR - mitigated the limitation of the light-receiving area associated with the increasing LAI (Fig. 3E), which is more likely to occur at a higher plant density. The dry matter production of plant stands per unit growing area, which is a product of the total DM per individual plant and plant density, was less under FR - than under $\mathrm{FR}+$ at all densities, and it increased as the plant density increased (Fig. 3F).

Photosynthate allocation. The leaf and root DM as a proportion of the total DM (i.e.,

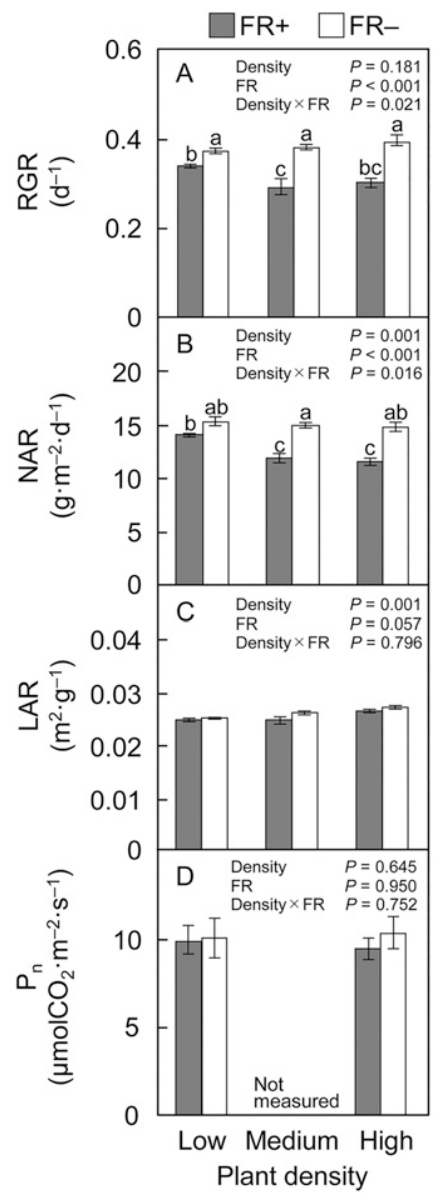

Fig. 4. Effects of interaction of plant density $x$ far-red (FR) treatment $(\mathrm{FR}+, \mathrm{FR}$ light similar to sunlight; FR-, no FR light) before transplanting on the (A) relative growth rate (RGR), (B) net assimilation rate (NAR), and (C) leaf area ratio (LAR) of cucumber seedlings after transplanting (10-14 d after seeding), and (D) net photosynthetic rate per unit leaf area $\left(\mathrm{P}_{\mathrm{n}}\right) 2 \mathrm{~d}$ after transplanting to pots (12 $\mathrm{d}$ after seeding). Data are the average $\pm \mathrm{SE}$ for seven replicates $(n=7)$, except for $P_{n}$ $(n=4)$. Bars for a parameter labeled with the same letter are not significantly different at $P<0.05$ (analysis of variance followed by the Tukey-Kramer test).

the leaf and root DM ratios) decreased with increasing plant density under FR+ (Fig. 3G and I), whereas that of stem DM increased (Fig. 3H). This is the typical response of shaded plants (Franklin, 2008). The lower allocation of photosynthate to the leaves may result from competition between the stems and leaves (Casal et al., 1987). However, under $\mathrm{FR}-$, the effect of plant density on the $\mathrm{DM}$ ratios was less than that under $\mathrm{FR}+$, and the stem and leaf DM ratios increased slightly and decreased, respectively, as the plant density increased (Fig. 3G-I). This is due to the decreased light competition under light with reduced FR.

Early growth after transplanting to pots. The RGR of seedlings after transplanting to pots was significantly less in the seedlings that had been grown under FR + at all plant densities and decreased at higher densities 


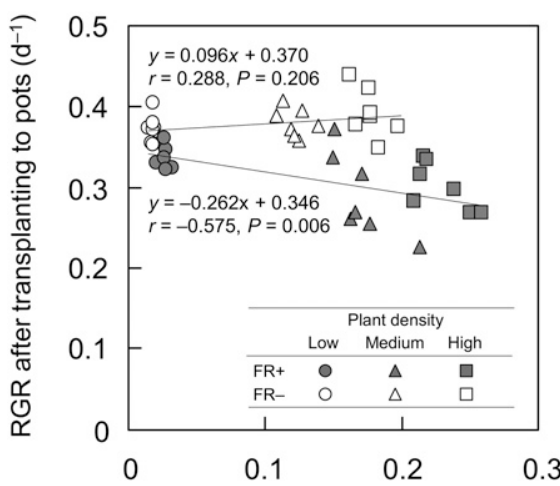

Dry matter production of plant stands $\left(\mathrm{kg} \cdot \mathrm{m}^{-2}\right)$

Fig. 5. Relationships between the dry matter production of plant stands per unit planting area at $4 \mathrm{~d}$ after the start of treatment (10 d after seeding) and relative growth rate (RGR) after transplanting to pots (10-14 d after seeding) for cucumber seedlings that had been grown under different combination of plant density and far-red (FR) treatment (FR+, FR light similar to sunlight; FR-, no FR light). The relationships between the variables were analyzed using Pearson's product-moment correlation coefficient $(r)$.

(Fig. 4A). However, the RGR of FR- was not significantly affected by plant density before transplanting. The RGR after transplanting in pots decreased as the dry matter production of plant stands per growing area increased under FR+ (Fig. 5), indicating that there are trade-offs between the productivity of transplants and subsequent early growth after transplanting plants with varying density. However, such trade-offs were not observed under FR- (Fig. 5). This means that the reduction in the quality of individual transplants due to growth at high plant density (Garner and Björkman, 1996) is less likely to occur under light with reduced FR.

The effect of the plant density $\times$ FR interaction before transplanting on RGR was significant, as was the case for NAR (Fig. 4B), whereas the interaction was not significant for LAR (Fig. 4C). The RGR and NAR after transplanting were strongly and significantly correlated with the photosynthate allocation, as measured by the leaf, stem, and root DM ratio at transplanting (Table 1); the seedlings that allocated less photosynthate to the leaves and root (more photosynthate to stem) tended to show lower RGR and NAR after transplanting.

Table 1. Relationships between the leaf, stem, and root dry mass (DM) as a proportion of total DM (the leaf, stem, and root DM ratios, respectively) at transplanting and the growth analysis parameters after transplanting.

\begin{tabular}{llll}
\hline \hline & \multicolumn{3}{c}{ Response valuable $(y)$} \\
\cline { 2 - 4 } Explanatory valuable $(x)$ & \multicolumn{1}{c}{ RGR } & \multicolumn{1}{c}{ NAR } & \multicolumn{1}{c}{ LAR } \\
\hline Leaf DM ratio & $y=0.557 x-0.530$ & $y=22.5 x-2.40$ & $y=-0.0004 x+0.0254$ \\
& $r=0.675^{* * *}$ & $r=0.750^{* * *}$ & $r=-0.016^{\mathrm{NS}}$ \\
Stem DM ratio & $y=-0.471 x+0.420$ & $y=-18.9 x+16.7$ & $y=-0.0001 x+0.0252$ \\
& $r=-0.720^{* * *}$ & $r=-0.792^{* * *}$ & $r=-0.005^{\mathrm{NS}}$ \\
Root DM ratio & $y=1.10 x+0.205$ & $y=43.0 x+8.24$ & $y=0.0030 x+0.0248$ \\
& $r=0.559^{*} * *$ & $r=0.599^{*} * *$ & $r=0.327^{\mathrm{NS}}$ \\
\hline
\end{tabular}

$\mathrm{RGR}=$ relative growth rate; $\mathrm{NAR}=$ net assimilation rate; $\mathrm{LAR}=$ leaf area ratio.

The units of variables are the same as those in Figs. 3 and 4.

*** Significant at $P<0.001$. NS $=$ nonsignificant at $P<0.05$
Ballaré, C.L., A.L. Scopel, and R.A. Sánchez. 1991. Photocontrol of stem elongation in plant neighbourhoods: Effects of photon fluence rate under natural conditions of radiation. Plant Cell Environ. 14:57-65.

Casal, J.J., P.J. Aphalo, and R.A., Sánchez. 1987. Phytochrome effects on leaf growth and chlorophyll content in Petunia axilaris. Plant Cell Environ. 10:509-514.

Franklin, K.A. 2008. Shade avoidance. New Phytol. 179:930-944.

Garner, L.C. and T. Björkman. 1996. Mechanical conditioning for controlling excessive elongation in tomato transplants: Sensitivity to dose, frequency, and timing of brushing. J. Amer. Soc. Hort. Sci. 121:894-900.

Hunt, R., D.R. Causton, B. Shipley, and A.P. Askew. 2002. A modern tool for classical plant growth analysis. Ann. Bot. 90:485-488.

Kono, M., H. Kawaguchi, N. Mizusawa, W. Yamori, Y. Suzuki, and I. Terashima. 2020. Far-red light accelerates photosynthesis in the low-light phases of fluctuating light. Plant Cell Physiol. 61:192-202.

Marr, C.W. and M. Jirak. 1990. Holding tomato transplants in plug trays. HortScience 25:173176.

Latimer, J.G. and C.A. Mitchell. 1988. Effects of mechanical stress or abscisic acid on growth, water status and leaf abscisic acid content of eggplant seedlings. Scientia Hort. 36:37-46.

Nagashima, H. and K. Hikosaka. 2011. Plants in a crowded stand regulate their height growth so as to maintain similar heights to neighbours even when they have potential advantages in height growth. Ann. Bot. 108:207-214.

Nagashima, H. and I. Terashima. 1995. Relationships between height, diameter and weight distributions of Chenopodium album plants in stands: Effects of dimension and allometry. Ann. Bot. 75:181-188.

Park, Y. and E.S. Runkle. 2017. Far-red radiation promotes growth of seedlings by increasing leaf expansion and whole-plant net assimilation Environ. Exp. Bot. 136:41-49.

Poorter, H., K.J. Niklas, P.B. Reich, J. Oleksyn, P. Poot, and L. Mommer. 2012. Photosynthate allocation to leaves, stems and roots: Metaanalyses of interspecific variation and environmental control. New Phytol. 193:30-50.

Radford, P.J. 1967. Growth analysis formulaetheir use and abuse. Crop Sci. 7:171-175.

Sager, J.C., W.O. Smith, J.L. Edwards, and K.L. Cyr. 1988. The use of spectral data to determine photosynthetic efficiency and phytochrome photoequilibria. Trans. Amer. Soc. Agr. Eng. 31:1882-1889.

Shibuya, T., S. Hayashi, R. Endo, and Y. Kitaya. 2016a. Growth analysis and photosynthesis measurements of cucumber seedlings grown under light with different red to far-red ratios. HortScience 51:843-846.

Shibuya, T., S. Kishigami, S. Takahashi, R. Endo, and Y. Kitaya. 2016b. Light competition within dense plant stands and their subsequent growth under illumination with different red:far-red ratios. Scientia Hort. 213:49-54.

Shibuya, T., S. Takahashi, R. Endo, and Y. Kitaya. 2013. Height-convergence pattern in dense plant stands is affected by red-to-far-red ratio of background illumination. Scientia Hort. 160:65-69.

Smith, H. and G.C. Whitelam. 1997. The shade avoidance syndrome: Multiple responses mediated by multiple phytochromes. Plant Cell Environ. 20:840-844.

Vandenbussche, F., W.H. Vriezen, J. Smalle L.J. Laarhoven, F.J. Harren, and D. Van Der 
Straeten. 2003. Ethylene and auxin control the Arabidopsis response to decreased light intensity. Plant Physiol. 133:517-527.

Vermeulen, P.J., N.P.R. Anten, F. Schieving, M.J.A. Werger, and H.J. During. 2008. Height convergence in response to neighbour growth: Genotypic differences in the stoloniferous plant Potentilla reptans. New Phytol. 177: 688-697.

Weiner, J. and S.C. Thomas. 1992. Competition and allometry in three species of annual plants. Ecology 73:648-656.

Zhen, S. and B. Bugbee. 2020. Far-red photons have equivalent efficiency to traditional photo- synthetic photons: Implications for redefining photosynthetically active radiation. Plant Cell Environ. 43:1259-1272.

Zhen, S. and M.W. van Iersel. 2017. Far-red light is needed for efficient photochemistry and photosynthesis. J. Plant Physiol. 209: 115-122. 